\title{
La evaluación previa de conocimientos favorece la actitud a la donación de órganos, independientemente de los argumentos ofrecidos
}

\section{Prior assessment of knowledge encourages organ donation attitude, independently of rationales offered}

\author{
Teresita Angélica López Ortiz, Susana Villalón Santillán \\ y Ferrán Padrós Blázquez ${ }^{1}$
}

Citación: López O., T.A., Villalón S., S. y Padrós B., F. (2019). La evaluación previa de conocimientos favorece la actitud a la donación de órganos, independientemente de los argumentos ofrecidos. Psicología y Salud, 29(2), 249-257.

\section{RESUMEN}

\begin{abstract}
Introducción: La creciente demanda de trasplantes entre la población plantea la necesidad de buscar y evaluar estrategias eficientes para fomentar actitudes favorables a la donación de órganos. Objetivo: Evaluar el efecto de diversas modalidades de ofrecer información en función del tipo de argumentos proporcionados y de la evaluación previa de las actitudes en favor de la donación en 137 estudiantes universitarios. Método: Mediante un enfoque cuantitativo de tipo comparativo, se llevaron a cabo tres modalidades de presentación: información sin argumentos, con argumentos en contra, o con argumentos a favor y en contra. A la muestra elegida se le aplicó la escala Actitud ante la Donación de Órganos de Morgan y Mille. Resultados: Se observó que la información proporcionada generó un cambio favorable de las actitudes en pro de la donación de órganos, independientemente de si se ofrecía sin ningún tipo de argumentos, explicitando los motivos para no donar, o con argumentos a favor y en contra de dicha donación. En cambio, se observó que una evaluación previa de las actitudes favorecía un cambio en favor de donar. Discusión: Hay una modificación favorable en las actitudes ante la donación de órganos y tejidos después de proporcionar la información; sin embargo, el ofrecimiento de argumentos en conjunto con la información proporcionada no parece influir en las actitudes de los jóvenes, y sí en cambio una evaluación previa.
\end{abstract}

Palabras clave: Obtención de tejidos y órganos; Actitud; Evaluación educacional; Trasplante de órganos.

\begin{abstract}
Introduction: The growing demand for organ transplants raises the need to seek and evaluate efficient strategies to encourage attitudes favorable to organ donation. Objective: Through a quantitative-comparative approach and along a pre-experimental design, the effect of various modalities of information and types of rationale were evaluated, including previous attitudes toward organ donation. Method: Three modalities of information were presented: information without rationale, with against rationale or with for and against rationales. A convenience sample divided into two groups replied to the Morgan and Mille Organ Donation Attitude Scale. One group was evaluated pre and post-intervention, and the other post-intervention only. Results: Information provided led
\end{abstract}

\footnotetext{
${ }^{1}$ Ciudad Universitaria, Universidad Michoacana de San Nicolás de Hidalgo, Av. Francisco J. Múgica s/n, tel. (443)322-35-00, 58030 Morelia, Mich., México, correos electrónicos: tanglica601_03@hotmail.com y susanavillalonsantillan@hotmail.com. Artículo recibido el 15 de marzo y aceptado el 21 de mayo de 2018.
} 
to a favorable attitude change toward organ donation, regardless of being offered without any rationale, with reasons not to donate, or with reasons for and against. The previous evaluation of attitudes comparison showed change in favor of donating. Discussion: There was a favorable change in attitudes toward organ and tissue donation after the information was provided. Rationale added to the information does not seem to affect attitudes of the participants, but a prior evaluation favors them.

Key words: Tissue and organ donation; Attitudes; Educational measurement; Organ transplantation.

\section{INTRODUCCIÓN}

$\mathrm{E}$ n sus más recientes estadísticas, el Centro Nacional de Trasplantes (COETRA) (2016) indica que 20,363 personas en México se encuentran en el registro nacional de espera. Es ésta una cifra alarmante, pues más de 20 mil mexicanos están en peligro de perder la vida de no conseguirse los órganos necesarios. Aunado a ello, se calcula que alrededor de $90 \%$ de esos pacientes morirá esperándolos.

Una posible estrategia para propiciar una actitud favorable de la población hacia la donación es utilizar técnicas persuasivas. En este caso, se concibe la persuasión como el logro mediante razones y argumentos (vía central) de que una persona actúe o piense de un modo determinado. Por consiguiente, ¿cuál es el efecto de diversas modalidades de ofrecer información sobre las mencionadas actitudes en jóvenes universitarios?, ¿tiene algún efecto la evaluación de las actitudes antes y después en la actitud favorable hacia la donación de órganos, o solo después de proporcionar información?, ¿influye la forma en que se ofrece la información sobre la donación en las actitudes de los jóvenes?

Es importante señalar que, en el estado de Michoacán (México), el COETRA reporta haber obtenido 616 órganos y tejidos entre los años 2003 y 2015 , producto de donaciones altruistas de connacionales, trasplantándose a 863 personas (Andrade y Gallegos, 2015).

Un área en la que el COETRA centra sus esfuerzos es la de difusión, con la cual proporciona a la población en general la información necesaria sobre la donación de órganos y tejidos a través de campañas y otras actividades. En su discurso, esas campañas resaltan la importancia de donar, lo que hacen utilizando eslóganes emotivos que se divulgan por los distintos medios de comunicación o en pláticas informativas en los diferentes niveles educativos (A.A., 2015; Ruiz, 2015). Con ello, el COETRA busca modificar las creencias erróneas que hay acerca de la donación de órganos entre la población y, por consiguiente, las actitudes de las personas sobre la misma (Ayala, 2006; Valdés, 1994).

La actitud se ha definido como el estado intrínseco de una persona que indica una tendencia a pensar, sentir, percibir y actuar respecto a un referente cognitivo, así como a realizar una evaluación favorable o desfavorable de una circunstancia, persona o hecho (Briñol, Horcajo y Petty, 2010; España, 2010; Huerta, 2008). Debe subrayarse que las actitudes han sido y son variables de considerable interés en el análisis del problema del rechazo a la donación de órganos y tejidos (Leal y Braga, 2008; Lozano, 2002).

Rossenberg y Hovland (1960) describen un modelo multidimensional de la actitud con tres componentes: el cognitivo, el afectivo-evaluativo y el actitudinal-conativo. El primero de ellos -el cognitivo- se denomina así porque hace referencia a los conocimientos adquiridos por medio de la experiencia que permiten hacer las evaluaciones de los objetos, personas o hechos. El componente afectivo-evaluativo se relaciona con las sensaciones placenteras o desagradables que provoca algún referente cognitivo $\mathrm{y}$, con ello, su valoración favorable o desfavorable. Por último, el componente actitudinal-conativo se relaciona con la motivación para la acción o reacción de la persona ante el objeto, persona o hecho en concreto (Arnau, Filella, Jariot y Montané, 2011; Díaz, 2002; Gutiérrez, 2009; Mora y Martinez, 2001; Torres y Fernández, 2012).

Cabe destacar que existen actitudes sólidas en las personas, quienes regulan la entrada y registro de nueva información ya sea ignorando o minimizando los datos que se oponen a la actitud de base, o bien atendiendo y recordando en mayor medida los que están en sintonía, lo que refuerza 
las actitudes previas. Con ello, las personas buscan -muchas veces de forma inconsciente- ser congruentes con sus actitudes previas. Por otro lado, algunas actitudes dominantes en una cultura o subcultura favorecen la identificación con los grupos de referencia. A aquellas que se inclinan hacia un punto extremo (positivo o negativo) se les califica como "polarizadas", y a las que no se asientan en un punto de vista claro y que cambian de un extremo a otro se les considera "ambivalentes" (Briñol, Falces y Becerra, 2007).

Las actitudes no son inamovibles; de hecho, nacen, se transforman y desaparecen; por ello, los estudiosos de la psicología social han tratado de diseñar estrategias para modificarlas (Moscovici, 2008). Las investigaciones buscan analizar el impacto en la actitud que tiene modificar algunos de sus componentes (cognitivo, emocional o conductual). Sí se quiere modificar una actitud, el componente indicado es el cognitivo, en cuanto que tiene preponderancia sobre los otros, por lo que la persuasión puede ser una herramienta para conseguir ese fin (Bernal, 2009), la que puede definirse como el fenómeno que tiene como propósito inducir a una persona a actuar de determinada manera o a adoptar alguna postura en particular generando ideas y haciendo que la aceptación de la información presentada se considere como verdadera por un actor externo que de modo consciente busca influir en las conductas y opiniones de aquélla mediante mensajes subliminales (Álvarez, 2010; Guerra, 2003; Moscovici, 2008; Simons, Morreale y Gronbeck, 2001; Tonani y Campos, 2008).

Se ha señalado que un mensaje persuasivo de gran impacto expresa argumentos en pro y en contra, pues de esa manera el receptor tiene la impresión de estar ante un emisor imparcial e íntegro; hasta cierto punto, la fuente puede catalogarse como creíble (Berrocal, 2004; Moscovici, 2008; Muñoz, 1994). Por otra parte, el mensaje puede ser enviado por diversos medios y su efecto se relaciona con la modalidad que se utilice (interpersonal, escrita o audiovisual), las interferencias y el doble flujo. En cuanto al receptor, sus características personales, la disposición que tenga a recibir la información, las variables sociodemográficas, su autoestima y la necesidad de aprobación, entre otras, pueden afectar el proceso persuasivo (Cavazza, 1999; Felicidad, 1998; Sabre, 2010a).
El modelo de probabilidad de elaboración (Elaboration Likelihood Model) (MPE en lo sucesivo) se centra en analizar la importancia que la motivación intrínseca tiene en las personas para elaborar un mensaje persuasivo que llegue a sus sentidos (Igartúa, 2011; Petty y Cacioppo, 1986; Sabre, 2010b). Al impactar el mensaje en el receptor, este puede analizar la información y aceptar o rechazar el mensaje. Lo interesante del proceso es entender hasta qué punto el sujeto puede elaborar cogniciones o pensamientos relacionados con el mensaje persuasivo recibido.

El proceso persuasivo implica dos rutas: la central y la periférica (Alvarado, 2011; Pastor y Juste, 2010; Pérez, 2011). La ruta central se activa cuando el sujeto presta atención a la información que se le transmite en virtud de que tiene interés en conocer sobre el tema; al captar el mensaje, analiza la información de manera detallada y sistemática, utilizando para ello las experiencias y conocimientos previamente adquiridos (Chávez, 2007; Falcez, Briñol, Sierra, Becerra y Alier, 2001). La ruta periférica se activa sobre todo cuando la persona tiene escaso interés en el mensaje y da respuestas rápidas guiadas por indicios simples, como el atractivo de la fuente, las recompensas, las características superficiales del mensaje, las reacciones de otros sujetos, el número de argumentos presentados y las estadísticas que lo avalan (Chávez, 2009; Moya, 1999).

El fenómeno de la persuasión, de acuerdo al MPE, ocurre de la siguiente manera: primeramente el mensaje persuasivo entra en contacto con el receptor, quien puede estar motivado o no para procesar la información. De estar motivado, entrará en un estado de interés para elaborar cogniciones, lo que activa el proceso de la ruta central, donde se analiza la calidad del argumento. Si se encuentra en disposición de descodificar la información persuasiva, el receptor elaborará pensamientos favorables, desfavorables o neutrales. Los pensamientos desfavorables y favorables provocan un cambio en la estructura cognitiva del destinatario que se puede identificar por medio de la modificación de las respuestas y cogniciones que aquél adopta y almacena en la memoria. De ser así, se puede propiciar un cambio de actitud positiva o negativa ante el mensaje (Hernández, 2009; NaiHwa, 2001; Pastor y Juste, 2010). 
En cuanto a la ruta periférica, lo primero que ocurre es la recepción del mensaje. El receptor, al no estar motivado para procesar la información, se centra en las variables relacionadas con aspectos que requieren poco análisis (afecto, credibilidad de la fuente y número de argumentos). Si cuenta con uno o más aspectos, podría haber una variación en la actitud con poca solidez y que predice escasamente la conducta (Chávez, 2009). La ruta central, en cambio, produce un cambio actitudinal más duradero y predice mejor la conducta (Briñol, Gandarillas, Horcajo y Becerra, 2010; Choi y Salmon, 2003; Falcez et al., 2001). Si se varía la información presentada y se muestran argumentos a favor, en contra o ambos, manteniendo como constantes la fuente y el contexto, es posible que varíe el grado de influencia de esa información en el receptor según sea el caso.

\section{Persuasión y donación de órganos}

En el ámbito de la donación de órganos y trasplantes, las campañas en las que se fomenta una actitud favorable hacia la misma utilizan diversos medios de transmisión (radio, televisión, prensa, conferencias, folletos, etc.) (Cornejo, Barril y Heredia, 2013). Investigaciones como la de Lozano (2002) y la de Díaz, Gautier, Pérez y Villarreal (2013) son ejemplos de estudios en los que se utilizan técnicas persuasivas para modificar esas actitudes. En el estudio de Lozano (2002), cuyo objetivo fue investigar las actitudes ante la donación de órganos y tejidos en una muestra de mexicanos, así como probar ciertas estrategias de persuasión, se dividió a la población en dos grupos: uno en el que un experto en el tema proporcionaba la información, y otro en el que los participantes la discutían, siendo el moderador el mismo experto. Al evaluar su impacto relativo en dichas actitudes y en la intención conductual (la elaboración del carnet de donador), se halló que la población estudiada tenía una actitud favorable hacia la donación de órganos y que, de las técnicas de persuasión utilizadas, la de mayor impacto fue aquella en que intervino el experto, pues modificó en un grado mayor las actitudes y la intención conductual. Díaz et al. (2013), pretendiendo comprobar si la presentación de argumentos positivos y negativos influyen en las actitudes hacia la donación de órganos, determinaron que no había diferencias estadísticamente significativas entre el grupo al que se le presentaron solamente argumentos en contra y el grupo que recibió únicamente argumentos a favor.

Debido al escaso número de estudios destinados a evaluar el efecto del cambio de actitudes de diferentes modalidades para ofrecer la información, el objetivo de la presente investigación fue estudiar si ofrecer información sobre la donación de órganos incide en las actitudes de los estudiantes, y analizar además si hay diferencias en el cambio de actitudes en función de diferentes modalidades: por un lado, según la evaluación de las actitudes (antes y después, o solo después de proporcionar información sobre la donación de órganos) y, por otro, según la forma en que se ofrece la información acerca de la donación de órganos, de la cual se consideraron tres modalidades: información base, información base con motivos para no donar, e información base con motivos para donar y para no hacerlo.

\section{Materiales}

Este fue un estudio cuantitativo de tipo comparativo, con un diseño experimental que tuvo como propósito principal evaluar el efecto de las diversas modalidades de información (en función del tipo de argumentos proporcionados y de si se evalúan previamente las actitudes) en las actitudes de estudiantes universitarios. Un primer objetivo específico fue verificar el impacto que tiene ofrecer información sobre las actitudes en los jóvenes. Uno más, determinar si hay un efecto en las actitudes en función de si éstas se evalúan antes de ofrecer la información. Por último, determinar si la modalidad de información proporcionada influye en las actitudes de los sujetos.

\section{MÉTODO}

\section{Participantes}

La muestra, elegida por conveniencia, fue de 137 participantes, todos ellos alumnos de las carreras de Administración de Empresas (23), Gestión Empresarial (93) e Ingeniería Electrónica (21) del Instituto Tecnológico Regional de Estudios Superio- 
res de Morelia (México). La muestra total estuvo conformada por 60 hombres $(43.8 \%)$ y 77 mujeres $(56.2 \%)$, con una edad media de 19.43 años (D.E. $=1.85)$. La población blanco se estableció tomando como criterio que fueran jóvenes de entre 18 a 23 años de edad.

\section{Instrumentos}

Para medir la actitud se utilizó el cuestionario Actitud ante la Donación de Órganos (Attitude Toward Organ Donation), en la versión de Morgan y Mi1le (2002). Es ésta una escala conformada por seis reactivos tipo Likert, cada uno de ellos con siete opciones de respuesta, que van de "Totalmente de acuerdo" a "Totalmente en desacuerdo". A mayor puntuación, el grado de actitud favorable ante la donación es más elevado. El cuestionario tiene una confiabilidad $\alpha$ de Cronbach de 0.88 .

\section{Procedimiento}

Primeramente, se contactó con los directivos y profesores del Instituto Tecnológico, a quienes se explicó el objetivo de la investigación y la importancia de su colaboración. Una vez que accedieron, se visitó a los alumnos para solicitarles su participación y obtener su consentimiento informado.

Las presentaciones se hicieron dentro de las instalaciones de la institución, en aulas ventiladas y con buena iluminación. De acuerdo a los objetivos de la investigación, primeramente se hizo un sorteo para determinar qué tipo de intervención iba a recibir cada grupo, y después se conformaron seis grupos en los que se combinaron los dos tipos de evaluación (preintervención, postintervención y solo postintervención) y las tres modalidades de presentación de la información del siguiente modo: Grupo 1: información básica y evaluación de la actitud antes y después de la intervención; Grupo 2: información básica junto con motivos en contra de donar y evaluación sólo después de la intervención; Grupo 3: información básica junto con motivos en contra de donar, y evaluación antes y después de la intervención; Grupo 4: Información básica junto con motivos a favor y en contra de donar, y evaluación sólo después de la intervención; Grupo 5: información básica junto con motivos a favor y en contra de donar, y evaluación antes y después de la intervención, y Grupo 6: información básica y evaluación de la actitud solo después de la intervención.

Las sesiones de los grupos evaluados antes y después tuvieron una duración aproximada de 45 minutos; en el caso de los grupos evaluados solamente después, esa duración fue de alrededor de 40 minutos. La diferencia de tiempo entre la exposición de la información base (30 minutos), información con los motivos para no donar (34 minutos) e información con los motivos para donar y no donar (36 minutos) fue de pocos minutos.

La denominada aquí "información base" contenía datos sobre qué es la donación, los tipos de donadores o el consentimiento familiar, entre otros. Por su parte, la información con motivos para donar incluía frases a favor, como "Darle la oportunidad a otra persona de mejorar su calidad de vida"; en contraste, los motivos para no donar mencionaban algunos posibles inconvenientes, como "Es un proceso largo que puede durar desde 24 hasta 72 horas".

\section{RESULTADOS}

Respecto al impacto del ofrecimiento de información sobre las actitudes, se observó después de la intervención (independientemente de la modalidad) una actitud más favorable $(\mathrm{M}=36.76$, D.E. $=4.0)$ hacia la donación $(\mathrm{t}[61]=-6.080 ; p<0.001)$ respecto a la media inicial $(\mathrm{M}=34.24$, D.E. $=5.03)$

Cuando se hizo la comparación entre las actitudes medidas antes y después de presentar la información y las que solo fueron evaluadas después, se observó que el nivel de las actitudes de los participantes que fueron evaluados previamente $(\mathrm{M}=36.76$, D.E. $=4.03)$ fue más favorable $(\mathrm{t}[132,249]=3.273 ; p=0.001)$ que el que manifestaron los participantes que solo fueron evaluados al finalizar la intervención $(\mathrm{M}=34.04$, D.E. $=5.66)$.

Sin embargo, no se observaron diferencias $(\mathrm{F}=1,653 ; p=0.195)$ en función del tipo de información proporcionada: información base $(\mathrm{M}=35.78$, D.E. $=4.65)$, información base con motivos para no donar $(\mathrm{M}=35.81$, D.E. $=4.51)$ e información base con motivos para no donar y para donar $(\mathrm{M}=34.07$, D.E. $=6.23)$. 
Por otro lado, cuando se realizó un ANOVA de dos vías, se observó un modelo significativo $(\mathrm{F}=3,319 ; p=0.007)$ con $\mathrm{R} 2=0.112$. Se observó significación respecto a si se hacía una evaluación previa $(\mathrm{F}=6,443.76 ; p=0.004)$, y no respecto a si la información se ofrecía sin argumentos, con motivos para no donar o con argumentos a favor $y$ en contra de donar $(\mathrm{F}=1,873.16, p=0.158)$. También resultó significativa $(\mathrm{F}=6,667.76, p=0.004)$ la intersección entre ambos factores (la evaluación de las actitudes pretest y postest y solamente postest y el tipo de información proporcionada). Se ofrecen los datos de cada grupo en la Tabla 1.

Tabla 1. Medias y desviaciones estándar de la actitud de los seis grupos evaluados antes y después.

\begin{tabular}{|l|c|c|c|c|c|}
\hline $\begin{array}{c}\text { Información } \\
\text { ofrecida }\end{array}$ & Grupos & \multicolumn{2}{|c|}{ Medición antes } & \multicolumn{2}{c|}{ Medición después } \\
\hline \multirow{2}{*}{ I B } & 1 & $\mathrm{M}=33.00$ & D.E. $=5.35$ & $\mathrm{M}=35.96$ & D.E. $=4.80$ \\
\cline { 2 - 6 } & 6 & & & $\mathrm{M}=35.50$ & D.E. $=4.55$ \\
\hline \multirow{2}{*}{ I + M C } & 2 & & & $\mathrm{M}=34.51$ & D.E. $=4.34$ \\
\cline { 2 - 6 } & 3 & $\mathrm{M}=36.10$ & D.E. $=4.21$ & $\mathrm{M}=38.10$ & D.E. $=3.91$ \\
\hline \multirow{2}{*}{ I + M F y C } & 4 & & & $\mathrm{M}=32.46$ & D.E. $=7.69$ \\
\cline { 2 - 6 } & 5 & $\mathrm{M}=33.72$ & D.E. $=5.14$ & $\mathrm{M}=36.22$ & D.E. $=2.67$ \\
\hline
\end{tabular}

Nota: I B: Información básica, I + M C: Información y motivos en contra I + M F y C: Información y motivos a favor y en contra.

\section{DISCUSIÓN}

El primer objetivo del presente estudio fue valorar el impacto de ofrecer información sobre las actitudes ante la donación de órganos y tejidos, corroborándose un cambio a favor de donar después de proporcionar la información (tomando en consideración las tres modalidades de información de forma conjunta), lo que concuerda con lo reportado por Díaz (2002). Por otro lado, respecto al segundo objetivo, se encontró que evaluar previamente las actitudes favoreció el cambio posterior, lo que podría explicarse en razón de que probablemente la actitud de la mayoría de los participantes antes de la intervención era favorable a la donación, es decir, polarizada al extremo positivo (Arenas, 2009). Así, la evaluación previa la hizo más presente y consciente, y es posible que después de la intervención identificaran algún otro elemento nuevo que fortaleciera la actitud identificada previamente.

En cuanto al tercer objetivo, que era determinar si la modalidad de información proporcionada influye en las actitudes de los jóvenes, no se observaron diferencias significativas en las tres modalidades consideradas (información básica, información básica junto con motivos para no donar e información básica junto con motivos para donar y no donar). El resultado discrepa de lo expuesto por Basanta y Romero (2010), quienes destacan la importancia de los argumentos bilaterales para mejorar la credibilidad y favorecer así el cambio de actitud, aunque debe señalarse que estos autores aluden a las actitudes de forma genérica. Sin embargo, en el ámbito de las actitudes respecto a la donación de órganos, Díaz et al. (2013) hallaron resultados similares, tal vez en virtud del hecho de que generalmente la mayoría de las personas tiene actitudes favorables hacia la donación de órganos, por lo que ofrecer argumentos no provoca resistencias en el receptor, como sucede en otros ámbitos (política, ofrecimiento de productos que pueden comprar, etc.), en los que el evaluado supone que se le puede estar manipulando (Briñol et al., 2010).

Un dato relevante es que el tema analizado fue al parecer de interés para los participantes, ya que algunos de ellos hicieron preguntas encaminadas a enriquecer la información obtenida, lo que podría estar relacionado con un proceso de elaboración de cogniciones detallado y, por tanto, con el uso de la ruta central (Chávez, 2009; Moya, 1999).

Es necesario tomar con cierta cautela los presentes resultados, pues deben señalarse algunas limitaciones del presente estudio. En primer lugar, no se conocen los datos de validez de la escala de 
actitud en población mexicana y tampoco de la versión original. También hay que recordar que el muestreo no fue representativo de la población; de hecho, la muestra empleada fue muy homogénea en cuanto a edad y formación, por lo que es necesario ser prudentes y no generalizar los hallazgos a población con características sociodemográficas diferentes. En posteriores investigaciones se sugiere realizar previamente estudios donde se determinen las propiedades psicométricas para la población mexicana de instrumentos sobre las actitudes hacia la donación de órganos.

Por otro lado, sería interesante realizar un seguimiento, de modo que se evaluara si el cambio de actitudes se mantiene en el tiempo, y asimismo analizar si el cambio supone algún tipo de acción observable, como por ejemplo hacerse del carnet de donante. Se propone ampliar la muestra con participantes de diferentes edades y niveles de escolaridad y, sobre todo, incluir personas que en principio manifiesten actitudes neutras o en contra de la donación de órganos, en los cuales es posible que el ofrecimiento de argumentos pueda ejercer una influencia significativa en su cambio. Además, parece recomendable que en in- vestigaciones subsecuentes se estudien aspectos sobre la credibilidad de la fuente (por ejemplo, el uso o no de bata blanca), así como el impacto que tiene el medio de trasmisión de la información en las actitudes.

Los resultados obtenidos indican que las campañas de donación de órganos en las que se proporciona meramente información, sin ofrecer ningún tipo de argumento a favor o en contra, son efectivas para favorecer la actitud hacia la donación de órganos, de modo que es más económico, y por ello más eficiente, proporcionar información sobre tal donación sin proveer argumentos adicionales al respecto. Se sugiere incidir sobre la población que tiene una actitud ya favorable hacia la donación (que probablemente es un porcentaje elevado) para aumentar el número de personas dispuestas a donar y, por tanto, disminuir la cantidad de pacientes que se encuentran a la espera de un trasplante. Los hallazgos de la presente investigación no desaconsejan que se hagan evaluaciones previas, al menos en personas que manifiestan una actitud favorable, debido a que parecen incidir de forma positiva sobre la actitud ante la donación de órganos.

\section{REFERENCIAS}

A.A. (2015). "Impulsan la cultura de donación de órganos”. La Voz de Michoacán, Sección Regional, 12 de octubre. Disponible en http://www.lavozdemichoacan.com.mx/regional/impulsan-la-cultura-de-donacion-de-organos/.

Alvarado, M.A. (2011). Influencia de la publicidad televisiva en el consumo de cereales azucarados en niños de Morelia. Tesis inédita de licenciatura. Morelia (México): Universidad Michoacana de San Nicolás de Hidalgo. Disponible en http://bibliotecavirtual.dgb.umich.mx:8083/jspui/handle/123456789/6618.

Álvarez, L. (2010). Modelos psicológicos del cambio: de los modelos centrados en el individuo a los modelos psicosociales en psicología de la salud. Psicología y Salud, 20(1), 97-102.

Andrade C., V. y Gallegos G., D. (2015). Nota informativa. Morelia (México): Consejo Estatal de Trasplantes del Estado de Michoacán.

Arenas S., M. (2009). Actitudes, percepciones y prácticas corporales de los usuarios adultos de programas de actividad física en las subregiones de Antioquia. Tesis inédita de maestría. Medellín (Colombia): Universidad de Antioquía. Disponible en: http://viref.udea.edu.co/contenido/pdf/145-actitudes.pdf.

Arnau S., L., Filella G., G., Jariot G., M. y Montané C., J. (2011). Evaluación de un programa de cambio de actitudes con preconductores para prevenir los accidentes de tráfico provocados por el alcohol en Cataluña. Adicciones, $23(3), 257-265$. Disponible en http://www.adicciones.es/ficha_art_new.php?art=728.

Ayala S., M. (2006). Donación y trasplante de órganos tejidos y células: mitos y realidades ( $2^{\mathrm{a}}$ ed). México: Trillas.

Basanta Z., G. y Romero P., R. (2010). Lineamientos teóricos de la comunicación persuasiva y su relación en la mediación de conflictos en Universidades Nacionales Experimentales del Estado de Zulia. Revista de Ciencias Sociales, 16(1), 28-38. Disponible en http://www.redalyc.org/articulo.oa?id=28016297004.pdf.

Berrocal, S. (2004). Una aproximación a la nueva retórica del líder político televisivo: acciones, cualidades y discurso. Doxa, 2, 53-67. Disponible en http://doxacomunicacion.es/pdf/artculoberrocal.pdf. 
Bernal S., A. (2009). Relación de las actitudes de los estudiantes hacia la matemática antes y después de haber cursado y aprobado los programas de cálculo diferencial e integral en la Universidad Sergio Arboleda. Tesis inédita de maestría. Bogotá (Colombia): Universidad Sergio Arboleda. Disponible en http://ima.usergioarboleda.edu.co/pelusa/pelusa(2)/documentos/ Tesis_Alejandro\%20Bernal.pdf.

Briñol, P., Falces, C. y Becerra, A. (2007). Actitudes, cambio de actitudes y proceso de influencia. En J. Morales., E. Gavira., M. Moya. e I. Cuadrado (Eds.): Psicología Social (pp. 458- 474). México: McGraw-Hill.

Briñol, P., Gandarillas, B., Horcajo, J. y Becerra, A. (2010). Emoción y meta-cognición: implicaciones para el cambio de actitud. Revista de Psicología Social, 25(2), 157-158. Disponible en http://www.uam.es/proyectosinv/attitude/Doc/Publicaciones/19_BRI\%C3\%91OL_ET_AL_2010.pdf.

Briñol, P., Horcajo, J. y Petty, R. (2010). Consumer persuasion: indirect change and implicit balance. Psychology and Marketing, 27(10), 938-963.

Cavazza, N. (1999). Comunicación y persuasión. Madrid: Acento Editorial.

Centro Nacional de Trasplantes (2016). Estadísticas. Morelia (México): COETRA. Disponible en http://www.cenatra.salud.gob. $\mathrm{mx} /$ interior/trasplante_estadisticas.html.

Chávez V., G. (2007). Estilos atribuciones y necesidad cognitiva en estudiantes universitarios del cuadro de méritos y de riesgo académico. Revista de Psicología, 9, 40-51. Disponible en http://sisbib.unmsm.edu.pe/BVRevistas/rev_psicologia_cv/ v09_2007/pdf/a04.pdf.

Chávez V., G. (2009). Estilos atribucionales, necesidades cognitiva y rendimiento académico en estudiantes universitarios. UCV Scientia, 1(1), 21-32. Disponible en http://revistas.concytec.gob.pe/pdf/scientia/v1n1/a14v1n1.pdf.

Choi, S.M. y Salmon, C. (2003). The elaboration likelihood model of persuasión after two decades: a review of criticisms and contributions. The Kentucky Journal of Comunication, 22(1), 47-77.

Cornejo C., G., Barril M., C. y Heredia J., F. (2013). Entrega de información y voluntariedad en el consentimiento presunto de la Ley de Donación de Órganos en Chile. Revista ANACEM, 7(1), 49-53. Disponible en http://revista.anacem.cl/web/wp-content/uploads/2013/07/7.1.13-ley-de-donaci\%C3\%B3n-de-\%C3\%B3rganos-en-Chile.pdf.

Díaz P., E. (2002). El factor actitudinal en la atención a la diversidad. Profesorado. Revista de Currículum y Formación del Profesorado, 6(1-2), 151-165. Disponible en http://www.ugr.es/ recfpro/rev61COL3.pdf.

Díaz, M., Gautier, R., Pérez, M. y Villarreal, J. (2013). Efecto del tipo de encuadre de resultados en los mensajes persuasivos sobre la actitud hacia la donación de órganos (Cartel). Jornada de Investigación en Psicología Experimental. Caracas: Universidad Central de Venezuela. Disponible en http://psicologiaucvdotcom.files.wordpress.com/2012/03/diaz-gauthier-perez-y-villareal.pdf.

España R., E. (2010). Conocimiento, actitudes, creencias y valores en los argumentos sobre un tema socio-científico relacionado con los alimentos. Tesis doctoral inédita. Málaga (España): Universidad de Málaga. Disponible en http://www.biblioteca. uma.es/bbldoc/tesisuma/17668566.pdf.

Falces, C., Briñol, P., Sierra, B., Becerra, A. y Alier, A. (2001). Validación de la Escala de Necesidad de Cognición y su aplicación al estudio del cambio de actitudes. Psicothema, 13(4), 622-628.

Felicidad L., A. (1998). De la persuasión a la intervención. Los MCM y su papel en la intervención comunitaria. Intervención Psicosocial, 7(3), 379-396. Disponible en http:/www.copmadrid.org/webcopm/publicaciones/social/43320.pdf.

Gutiérrez T., D. (2009). Las actitudes de los residentes ante el turismo. Tesis doctoral inédita. Santa Cruz de Tenerife (España): Universidad de la Laguna. Disponible en ftp://tesis.bbtk.ull.es/ccssyhum/cs256.pdf.

Guerra G., A. (2003). Comunicación persuasiva en las vallas de licores y bebidas alcohólicas en el Municipio de Guatemala. Tesis inédita de licenciatura. Guatemala: Universidad Francisco Marroquín. Disponible en http://www.tesis.ufm.edu.gt/ pdf/3751.pdf.

Hernández P., M. (2009). Efectos de la manipulación del arousal /valencia de programas de televisión en bloques publicitarios: estudios psicofisiológicos. Tesina inédita de de licenciatura. Murcia (España) Universidad de Murcia. Disponible en http:// digitum.um.es/xmlui/bitstream/10201/19377/1/Tesina\%20de\%20Licenciatura\%20de\%20Manuel\%20Hernandez\%20 Perez\%202009.pdf.

Huerta P., J. (2008). Actitudes humanas, actitudes sociales. Madrid: Ministerio del Trabajo y Asuntos Sociales. Disponible en http://umer.es/wp-content/uploads/2015/05/n47.pdf.

Igartúa J., J. (2011). Mejor convencer entreteniendo: comunicación para la salud y persuasión narrativa. Revista de Comunicación y Salud, 1(1), 69-83. Disponible en http://www.revistadecomunicacionysalud.org/index.php/rcys/article/view/12.

Leal M., E. y Braga M., M. (2008). El rechazo al pedido de donación de órganos y tejidos para transplante. Revista Latinoamericana Enfermagem, 16(3), 1-8.

Lozano R., G. (2002). Actitudes y estrategias de persuasión hacia la donación de órganos y tejidos. Tesis inédita de doctorado. México: Universidad Nacional Autónoma de México. Disponible en http://132.248.9.195/pdtestdf/0309346/Index.html.

Mora R., J. y Martínez S., J. (2001). Escala de Actitud hacia la Homosexualidad: propiedades psicométricas y aspectos diferenciales por sexos. Revista Internacional de Ciencias Sociales y Humanidades, 21(1), 105-124. Disponible en http://www. redalyc.org/pdf/654/65421407006.pdf. 
Morgan, S.E. y Miller, J.K. (2002). Communicating about gifts of life: The effect of knowledge, attitudes, and altruism on behavior and behavioral intentions regarding organ donation. Journal of Applied Communication Research, 30, $163-178$.

Moscovici, S. (2008). Psicología social I: Influencia y cambio de actitudes, individuos y grupos (3ª ed.). México: Paidós.

Muñoz, B. (1994). El estado actual de la sociología de la cultura y de la comunicación de masas en los estados. Revista de la Facultad de Educación de Albacete, 8, 95-109. Disponible en https://dialnet.unirioja.es/descarga/articulo/2282505.pdf.

Moya, M. (1999). Persuasión y cambio de actitudes. En J. F. Morales (Ed.): Psicología social (2a ed.) (pp. 215-232). Madrid: McGraw-Hill.

Nai-Hwa, L. (2001). Elaboration likelihood model in costumer research: a review. Proceedings of the National Science Council, 11(4), 301-310.

Pastor, L. y Juste, J. (2010). Persuasión bajo la línea de flotación. Investigaciones sobre la vía heurística en los medios de comunicación. Análisis, 40, 47-67. Disponible en ///C:/Users/admin/Downloads/243375-326375-1-PB\%20(4).pdf.

Pérez R., I. (2011). Estudio de la imagen de responsabilidad social corporativa: formación e integración en el comportamiento del usuario de servicios financieros. Tesis inédita de doctorado. Santander (España): Universidad de Cantabria. Disponible en http://www.tdx.cat/bitstream/handle/10803/36868/0de5.APRprevio.pdf?sequence=1.

Petty, R.E. y Cacioppo, J.T. (1986). Elaboration Likelihood Model. Advances in Experimental Social Psychology, 19, $124-162$.

Rosenberg, M.J. y Hovland, C.L. (1960). Cognitive, affective, and behavioral components of attitudes. En C.I. Hovland y M. J. Rosenberg (Eds.): Attitude organization and change: An analysis of consistency among attitude components (pp. 1-14). New Haven, CT: Yale University Press.

Ruiz, C. (2015). Aumenta la cultura de la donación de órganos en Michoacán. Quadrantín. Disponible en https://www.quadratin. com.mx/morelia/Aumenta-la-cultura-donacion-organos-Michoacan/.

Sabre, M. (2010a). Implicación y encuadre como factores de eficacia en los mensajes publicitarios de las ONG. Comunicación y Sociedad, 24(1), 269-301.

Sabre, M. (2010b). Publicidad audiovisual de las organizaciones no gubernamentales de desarrollo (ONGD). El encuadre y la implicación como factores de eficacia persuasiva. Tesis inédita de doctorado. Salamanca (España): Universidad de Salamanca. España. Disponible en http://gredos.usal.es/jspui/bitstream/10366/76553/1/DSC_ElisaSabreM_PublicidadAudiovisualONGD.pdf.

Simons, H., Morreale, J. y Gronbeck, B. (2001). Persuasion in society (2nd ed.). Thousand Oaks, CA: Sage.

Tonani, M. y Campos, C.E. (2008). Cancer risk and preventive behavior: persuasion as an intervention strategy. Revista Latinoamericana Enfermagen, 16(5), 864-870. Disponible en http:/www.scielo.br/scielo.php?pid=S0104-11692008000500011\&script $=$ sci_arttext.

Torres R., E. y Fernández C., A. (2012). Instrumento para el análisis y evaluación de los conocimientos, actitudes y acciones hacia los murciélagos en la Mixteca poblana. Investigación Ambiental, 4(1), 4-18. Disponible en http://www2.inecc.gob. $\mathrm{mx} /$ publicaciones/gacetas/663/murcielagos.pdf.

Valdés D., M. (1994). Estudio de las actitudes ante la muerte en cónyuges de pacientes hospitalizados en unidades de cuidados intensivos generales. Tesis inédita de doctorado. Sevilla (España): Universidad de Sevilla. Disponible en http://www.psiquiatria.com/imgdb/archivo_doc9003.pdf. 